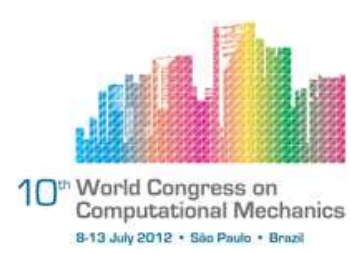

\title{
COMPRESSIBILITY EFFECTS ON AIRFOIL TRAILING EDGE NOISE GENERATION AND PROPAGATION
}

\author{
W. R. Wolf ${ }^{1,2}$, J. L. F. Azevedo ${ }^{2}$, S. K. Lele ${ }^{1,3}$ \\ ${ }^{1}$ Department of Aeronautics \& Astronautics, Stanford University (willwolf@ stanford.edu) \\ ${ }^{2}$ Instituto de Aeronáutica e Espaço, IAE/DCTA \\ ${ }^{3}$ Department of Mechanical Engineering, Stanford University
}

\begin{abstract}
The present investigation of airfoil trailing edge noise concerns the broadband noise that arises from the interaction of turbulent boundary layers with the airfoil trailing edge and the tonal noise that arises from vortex shedding generated by laminar boundary layers. We address aspects of noise generation and propagation such as the effects of mean flow convection on the calculation of sound directivity, of compressibility and quadrupole sources on sound radiation. Large eddy simulations (LES) are conducted for a NACA0012 airfoil with rounded trailing edge for two flow configurations with different freestream Mach numbers $\left(M_{\infty}=0.115\right.$ and 0.4$)$ for an angle of incidence AoA $=5$ deg. The Reynolds number based on the airfoil chord is fixed at $R e_{c}=408000$. The acoustic predictions are performed by the Ffowcs Williams \& Hawkings (FWH) acoustic analogy formulation and incorporate convective effects. Aeroacoustic results are compared to experimental data for a case which presents a broad vortex shedding tone and good agreement is observed.
\end{abstract}

Keywords: Airfoil noise, LES, Acoustic analogy, Broadband noise, Tonal noise.

\section{INTRODUCTION}

The understanding of trailing edge noise generation and propagation is of paramount importance for the design of low-noise aerodynamic shapes such as wings and high-lift devices, as well as wind turbine blades, propellers and fans. Airfoil trailing edge noise may originate from laminar and turbulent boundary layers, bluntness of the trailing edge and flow separation/stall [1]. Several authors have recently investigated the problem of trailing edge noise generation and propagation using numerical simulations $[2,3,4,5,6,7,8]$. However, many previous studies of airfoil noise used incompressible LES. Such approach restricted the application of the unsteady surface pressures, directly computed by LES, to acoustic analogy formulations only in the low frequency regime. Other limitations of some previous investigations include insufficient near-wall resolution issues for the meshes used, application of low-order schemes and insufficient domain sizes. 
In the present investigation, we apply a high-order accurate non-dissipative compact scheme implemented on a staggered grid together with an overset grid method that uses highorder accurate Hermite interpolation between grid blocks. The capability to achieve good grid quality along the airfoil surface is of paramount importance for accurately capturing the flow physics. Such accurate solution is given as input to a propagation model, which is, then, used to calculate the far field sound signature. In this study, compressible LES is used to obtain accurate wall pressure data that are used in the FWH acoustic analogy formulation for all frequency ranges.

The primary concern of this study is the broadband noise that arises from the interaction of turbulent boundary layers with the airfoil trailing edge and the tonal noise that arises from vortex shedding generated by laminar boundary layers. Large eddy simulations (LES) are conducted for a NACA0012 airfoil with rounded trailing edge for two flow configurations with different freestream Mach numbers $\left(M_{\infty}=0.115\right.$ and 0.4$)$ for an angle of incidence $\mathrm{AoA}=5 \mathrm{deg}$. The Reynolds number based on the airfoil chord is fixed at $R e_{c}=408000$. We address aspects of noise generation and propagation such as the effects of mean flow convection on the calculation of sound directivity, of compressibility and quadrupole sources on sound radiation. The acoustic predictions are performed by the FWH acoustic analogy formulation [9] and incorporate convective effects. The surface and volume integrations of dipole and quadrupole source terms appearing in the FWH equation are performed using the 3D convective wideband multi-level adaptive fast multipole method (FMM) developed in [10, 11, 12] to reduce the computational cost of the calculation of aeroacoustic integrals in the FWH formulation. With the method applied in this work the computational cost of evaluating the aeroacoustic integrals is considerably reduced.

The far field sound spectrum computed for the lower freestream Mach number configuration, $M_{\infty}=0.115$, is compared to experimental data by Brooks et al. [1] and excellent agreement is observed. Non-linear quadrupole noise sources play an important role in far field sound radiation for the higher Mach number flow configuration, $M_{\infty}=0.4$. A scaling study is performed for surface pressure spectra and good hydrodynamic scaling is observed.

\section{FLOW SIMULATIONS AND ACOUSTIC PREDICTIONS}

The general curvilinear form of the compressible Navier Stokes equations is solved using LES. The numerical scheme for spatial discretization is a sixth-order accurate compact scheme [13] implemented on a staggered grid. The current numerical capability allows the use of overset grids with a fourth-order accurate Hermite interpolation between grid blocks. A detailed explanation of the overset grid capability including implementation, verification and validation aspects can be found in [14]. In this reference, the overset procedure was applied for the solution of aerodynamic and aeroacoustic problems. The time integration of the fluid equations is carried out by a fully implicit second-order Beam-Warming scheme [15] in the near-wall region in order to overcome the time step restriction. A third-order Runge-Kutta scheme is used for time advancement of the equations in flow regions far away from solid boundaries. No-slip adiabatic wall boundary conditions are applied along the solid surfaces except for the tripping region where suction and blowing is applied. Characteristic plus sponge boundary conditions are applied in the far field locations and periodic boundary 
conditions are applied in the spanwise direction. The dynamic subgrid model formulation of Lilly [16] is used to include the effects of unresolved turbulent scales. The numerical tool has been previously validated for several compressible flow simulations including aeroacoustic applications [12, 14, 17].

The Ffowcs Williams \& Hawkings [9] (FWH) acoustic analogy is used for the acoustic predictions. A three-dimensional boundary integral formulation of the FWH equation that includes convective effects is implemented in the frequency domain [18]. The surface integrations appearing in the FWH equation are computed along the scattering body surfaces. Therefore, the monopole source terms are steady in time and do not appear in the frequency domain formulation and the dipole source terms are given only by the unsteady surface pressure. One should mention that viscous effects are neglected for the problems analyzed. For the current Mach numbers analyzed, the quadrupole sources are given only by the Reynolds stresses since entropy variation effects are negligible. The integrations of these volumetric sources are computed along a subset region of the flowfield including the wake plus turbulent boundary layer regions, where the magnitude of non-linear sources is non-negligible. The surface and volume integrations of dipole and quadrupole source terms are performed using a $3 \mathrm{D}$ convective wideband multi-level adaptive FMM $[10,11,12]$ to reduce the computational cost of the calculation of aeroacoustic integrals in the FWH formulation. The developed numerical capability allows the analysis of each noise source individually as well as the effects of convection on the computation of noise radiated by these sources. Therefore, it is possible to investigate the effects of dipole and quadrupole sources for each configuration.

\section{RESULTS}

The present investigation allows a study of sound generated by turbulent and laminar boundary layers that develop along the suction and pressure sides of a NACA0012 airfoil at five deg. angle of incidence. The flow Reynolds number based on the airfoil chord is set $R e_{c}=408000$ and the freestream Mach numbers are $M_{\infty}=0.115$ and 0.4. For both configurations, the boundary layer is tripped on the suction side of the airfoil by suction over the region $0.15<x / c<0.175$ and blowing over the region $0.175<x / c<0.20$. This tripping zone is chosen to model the experimental tripping used by Brooks et al. [1] for a similar flow configuration at $M_{\infty}=0.115$. The magnitude of suction and blowing is constant over $0.01<z / c<0.09$ with $\left|U_{\text {blowing }}\right|=\left|U_{\text {suction }}\right|=0.03 U_{\infty}$ chosen from numerical experimentation.

The grid configuration consists of a body-fitted O-grid block of size $960 \times 125 \times 128$ that accurately resolves the turbulent and laminar boundary layers and a Cartesian background grid block of size $896 \times 511 \times 64$ with uniform resolution around the O-grid block and that gently stretches up to the far field regions. In Figs. 1 (a) and (b) one can see the full view of the computational grid with approximately 45,000, 000 grid points and a detail of the Ogrid around the airfoil region, respectively. Every $4-t h$ grid point is shown in these figures. Figure 1 (c) shows a detail view of the blunted trailing edge. The spanwise width is $L_{Z}=0.1 \mathrm{c}$ and the mesh distribution along the airfoil span is uniform with $\Delta z=0.1 c / 128$. The grid distribution over the airfoil is not symmetric and more points are distributed over the suction side of the airfoil to have higher resolution. The overset grids are carefully designed to capture 
the turbulent and laminar boundary layers plus wake at an affordable computational cost. Maximum values of grid spacing in terms of wall units for both test cases analyzed are given by $\Delta x^{+} \approx 60, \Delta y^{+} \approx 0.5$ and $\Delta z^{+} \approx 20$ and occur at the transition region at $x / c \approx 0.3$. At the trailing edge region, $0.8<x / c<1.0$, values of grid spacing in terms of wall units are given by $\Delta x^{+} \approx 10, \Delta y^{+} \approx 0.3$ and $\Delta z^{+} \approx 10$. For the present grid configuration, span-averaged values of $\mu_{S G S}$ along the airfoil turbulent boundary layer reach peak values of $\mu_{S G S} \approx 0.1 \mu_{\infty}$ and, along the laminar boundary layer region, $\mu_{S G S}=0$.

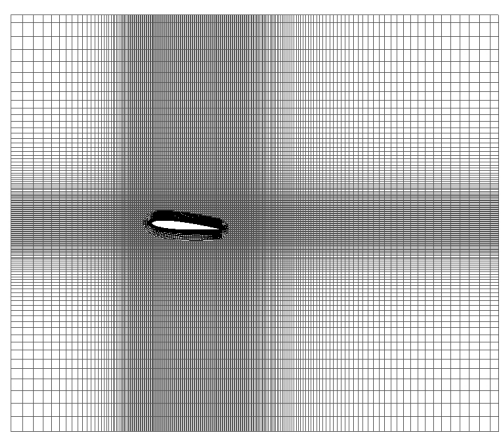

(a) Full view of computational grid (every $4-t h$ grid point shown).



(b) Detail view of O-grid around airfoil region (every $4-t h$ grid point shown).

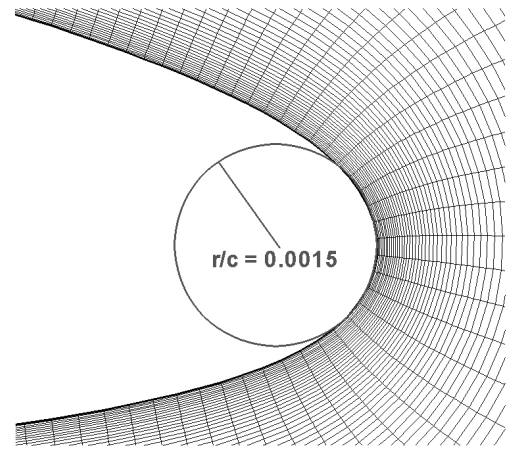

(c) Detail view of the rounded trailing edge.

Figure 1. Mesh details for large eddy simulation of flow past a NACA0012 airfoil at $\alpha=5$ deg. and $R e_{c}=408000$.

The three-dimensional simulations are started from spanwise extruded 2D solutions that are computed until all the transients vanish to ensure smooth 3D acoustic fields. Figures 2 (a) and (b) show iso-surfaces of vorticity magnitude colored by streamwise momentum and a background slice with dilatation contours in gray scale for the $M_{\infty}=0.115$ and 0.4 configurations, respectively. Although turbulent boundary layer and wake structures are similar for both figures, one can see that higher frequencies are present in the dilatation contours shown in Fig. 2 (b) for the higher freestream Mach number case. It appears from Fig. 2 that the steady blowing and suction does not generate any spurious noise, which certainly would be in contrast with the physics obtained if unsteady suction and blowing were used. This observation is drawn from inspection of the dilatation field in the cited figure and other similar visualizations shown in the present work. However, it is clear that further studies of the effect of using steady blowing/suction for boundary layer tripping would be interesting, but these are beyond the scope of the present investigation.

In Figs. 3 (a) and (b), one can observe plots of wall pressure power spectral densities in dimensional units and normalized by outer variables, respectively, obtained by the present LES at $x / c=0.95$ on the suction and pressure sides of the airfoil. Results normalized with outer variables use $q_{e}=1 / 2 \rho U_{e}^{2}$ as the pressure scale and $\delta^{*} / U_{e}$ as the time scale. Here, $U_{e}$ is the velocity at the edge of the boundary layer and $\delta^{*}$ is the boundary layer displacement thickness. In Fig. 3 (b), one can see a narrow band peak at non-dimensional frequency $\omega \delta^{*} / U_{e} \approx 0.55$ (Helmholtz numbers $k c \approx 9$ and 27 for $M_{\infty}=0.115$ and 0.4 , respectively) due to vortex shedding from the laminar boundary layer. Figure 3 (a) presents similar results using dimensional values of wall pressure PSDs in order to demonstrate that the vortex shed- 


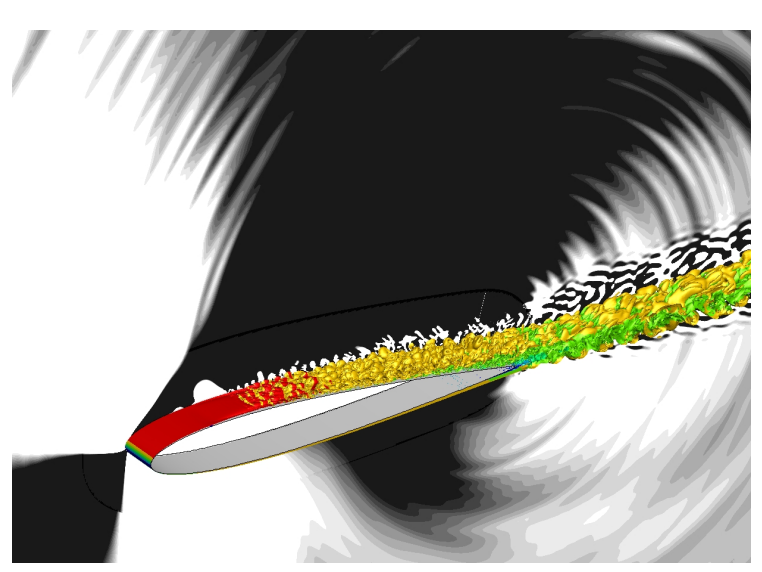

(a) $M_{\infty}=0.115$

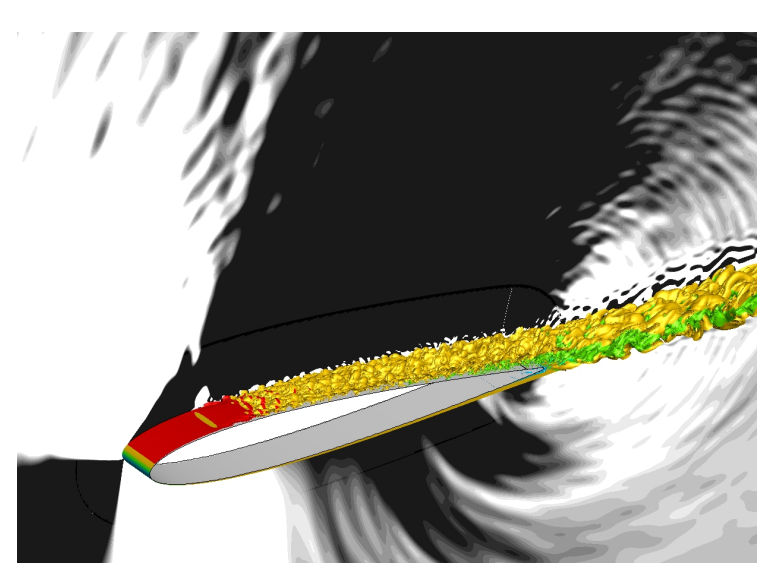

(b) $M_{\infty}=0.4$

Figure 2. Iso-surfaces of vorticity magnitude colored by streamwise momentum with contours of dilatation in the background.

ding energy levels are lower compared to those observed in the suction side of the boundary layer. The results demonstrate that the normalization is effective in collapsing the wall pressure PSD curves. This means that surface pressure spectra scale with hydrodynamic scaling, as suggested in [19]. The results also show that the normalized spectra do not present any significant differences due to the changes in freestream Mach number.

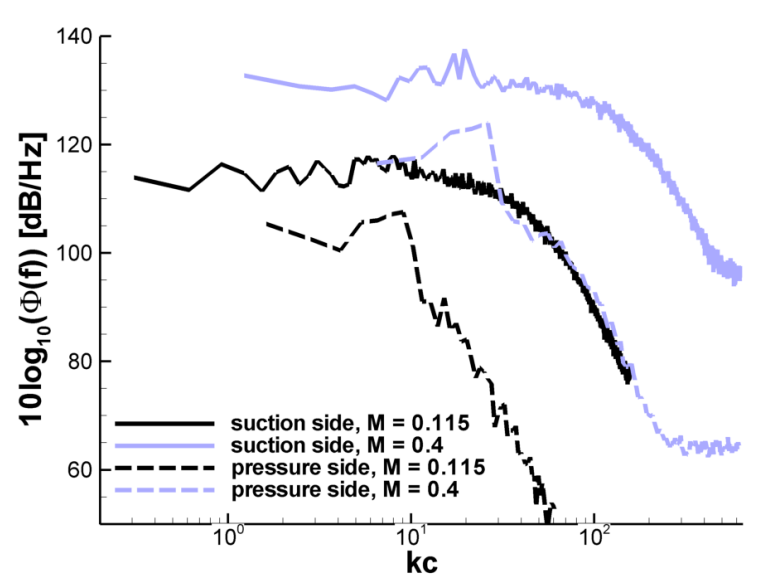

(a) Dimensional spectra.

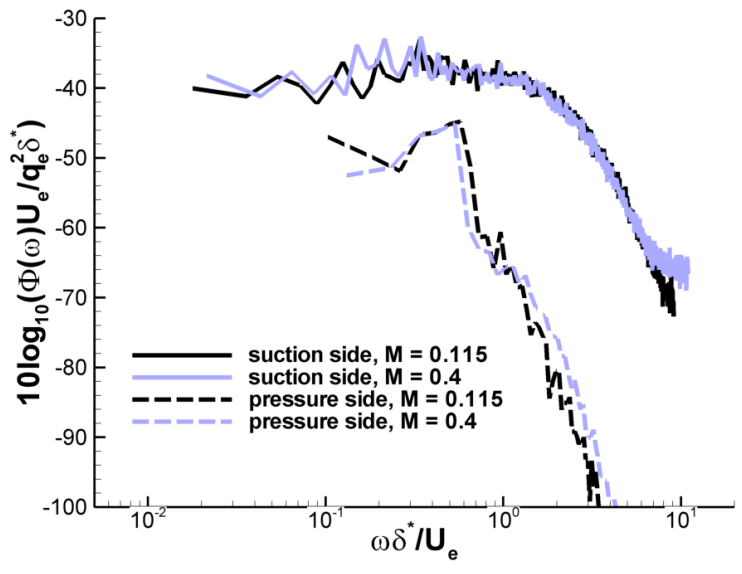

(b) Normalization by outer variables.

Figure 3. Wall pressure power spectral densities at $x / c=0.95$.

Figures 4 (a) and (b) present plots of spanwise vorticity along the airfoil and wake regions for both LES and, as one can see, confirms the presence of the vortex shedding. In Figs. 4 (c) and (d), one can see streamlines formed by the mean velocity showing a laminar boundary layer separation on the pressure surface of the airfoil prior to trailing edge, near $x / c=0.9$, and a recirculation bubble that forms for both configurations.

The FWH acoustic analogy formulation is used to predict the far field sound generated by the airfoil and the present LES provides the near flowfield used to compute the acoustic source terms that are transformed to the frequency domain. Dipole source integrations are computed along the airfoil surface. The effects of quadrupole sources are found to be negligible for the $M_{\infty}=0.115$ configuration as shown in [20] and, therefore, are not included 


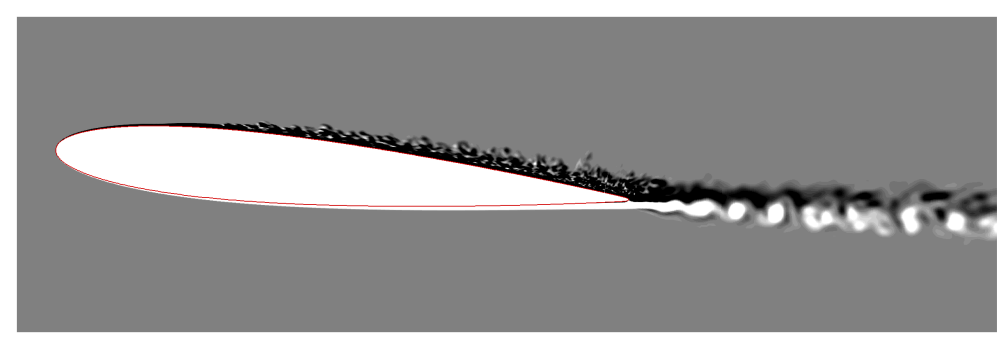

(a) $M_{\infty}=0.115$.

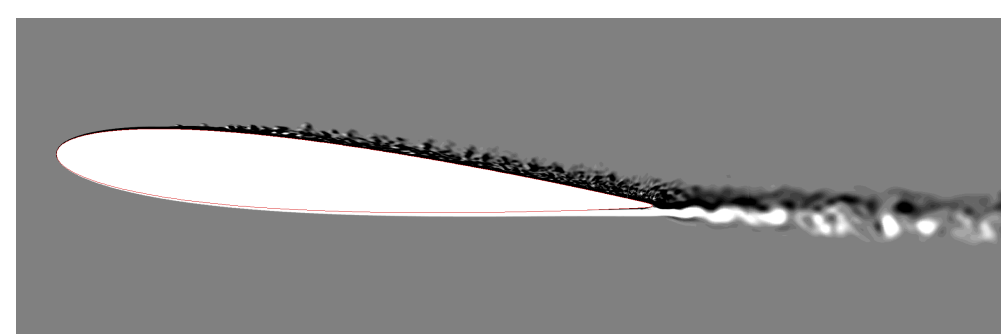

(b) $M_{\infty}=0.4$

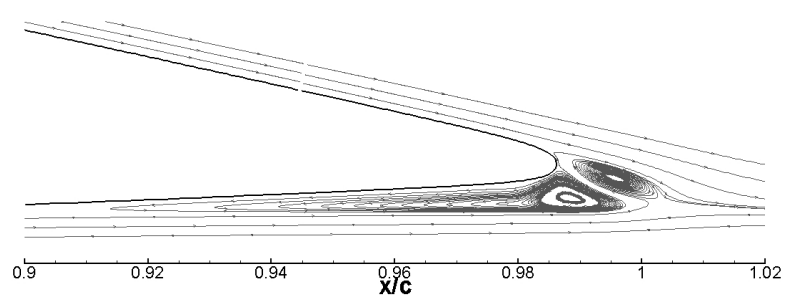

(c) $M_{\infty}=0.115$

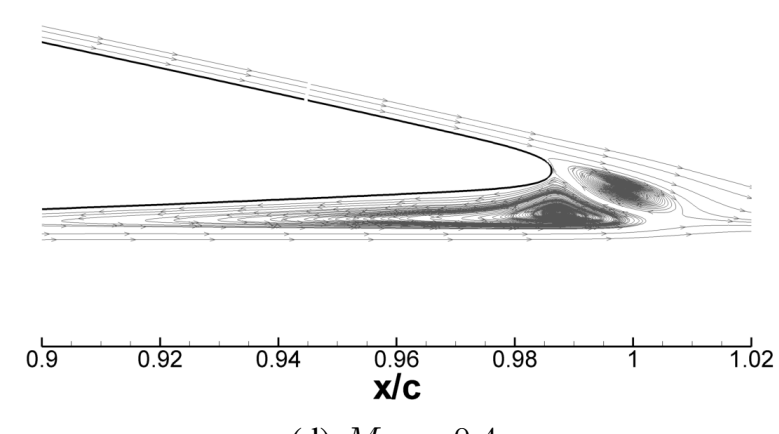

(d) $M_{\infty}=0.4$

Figure 4. Spanwise vorticity along airfoil and wake regions (a) and (b) and enlarged view of mean flow streamlines along trailing edge region (c) and (d). 
in the acoustic prediction for this case. However, the acoustic prediction for the $M_{\infty}=0.4$ configuration includes the contributions of both dipoles and quadrupoles, since, in this case, one expects that the volumetric sources should play a significant role in the sound generation. The frequency domain source terms are computed from 768 and 1024 time samples divided in five segments of 256 records and three segments of 512 records for the lower and higher Mach number cases, respectively. Calculations use $50 \%$ overlap of data samples. The LES calculations are performed using non-dimensional time steps of $\Delta t=0.0004$ for the $M_{\infty}=0.115$ case and 0.0001 for the $M_{\infty}=0.4$ in order to have accurate sampling of solutions for Fourier analysis. Before collecting data, the simulations are advanced in time over 10 and 15 airfoil flow-through times, respectively, and data is collected over 2 airfoil flow-through times.

Numerical results obtained for the $M_{\infty}=0.115$ configuration are compared to experimental data provided by Brooks et al. [1]. The experiments were conducted in a lowturbulence open rectangular jet with exit dimensions given by $30.48 \times 45.72 \mathrm{~cm}$ located in an anechoic chamber. The airfoil chord and span used in the experiment were 15.24 and 45.72 $\mathrm{cm}$, respectively. More details regarding the experimental setup can be found in Ref. [1]. In the experiments, the flow Reynolds and Mach numbers are identical to those used in the LES calculation. However, in the experiment, the airfoil trailing edge is sharp and its angle of incidence is AoA $=10.8 \mathrm{deg}$. One should mention that flow curvature and downwash deflection are different in the wind tunnel experiment and in an ideal undisturbed freestream flow. Brooks et al. [1] used lifting surface theory to develop a wind tunnel correction to the airfoil angle of incidence. An airfoil with the corrected angle of incidence in an undisturbed freestream should provide the same lift as an airfoil with the actual wind tunnel angle of incidence. For the present configuration, the corrected angle of incidence found by Brooks et al. is $\mathrm{AoA}=5.4 \mathrm{deg}$.

In the experiment, the spanwise width is three times the chord length, $L_{Z-E X P}=$ $3 c$, and, in the present LES, the spanwise width is $L_{Z-L E S}=0.1 c$. Therefore, the ratio $L_{Z-E X P} / L_{Z-L E S}=30$. An assessment of the spanwise coherence is necessary to predict the frequency spectrum of the sound pressure radiated by the full span width used in the experiment. The pressure spanwise coherence is defined as

$$
\gamma^{2}(z, \Delta z, f)=\frac{\left|\Phi_{p p}(z, \Delta z, f)\right|^{2}}{\left|\Phi_{p p}(z, 0, f)\right|\left|\Phi_{p p}(z+\Delta z, 0, f)\right|},
$$

where the cross spectrum function $\Phi_{p p}$ is the Fourier transform of the space-time cross correlation function

$$
\Phi_{p p}(z, \Delta z, f)=\int_{-\infty}^{\infty}\langle p(z, t) p(z+\Delta z, t+\tau)\rangle e^{-i f \tau} d \tau .
$$

Figure 5 shows the spanwise coherence of the surface pressure on the upper surface of the airfoil at $x / c=0.95$ for three different frequencies and on the lower surface of the airfoil at $x / c=0.985$ for the vortex shedding frequency. As one can notice, the coherence drops considerably for the high frequencies on the upper surface of the airfoil and, for these frequencies, source regions separated by $L_{Z-L E S}$ radiate sound independently from neighboring sources in a statistical sense. Therefore, the total noise spectrum is computed as the sum of contributions from $L_{Z-E X P} / L_{Z-L E S}$ independent source regions along the span [21]. The 
drop for the vortex shedding frequency is less pronounced as one can see in the plot. However, frequencies just below and above this frequency show a considerable drop in the value of coherence.

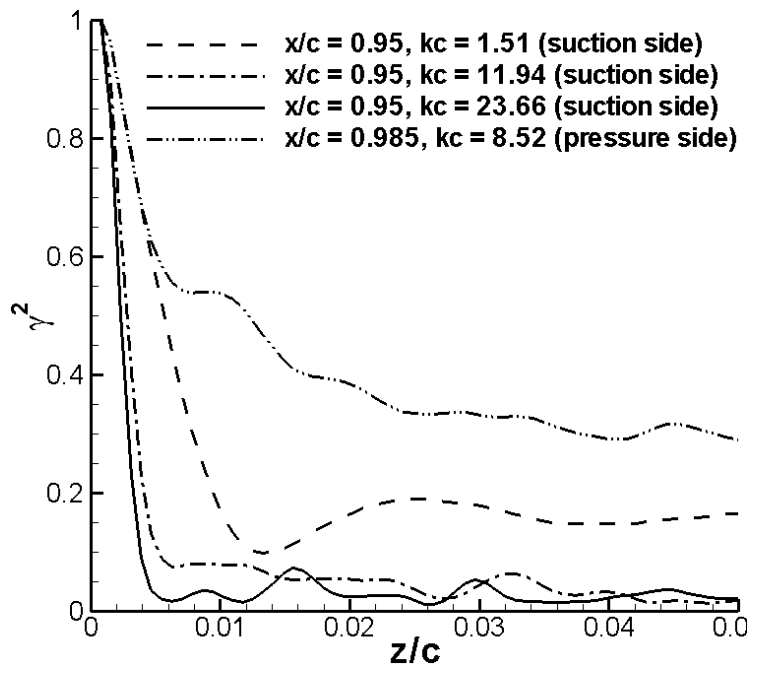

Figure 5. Spanwise coherence of surface pressure for the $M_{\infty}=0.115$ test case.

In Fig. 6, one can observe a comparison of sound pressure level between the current prediction and experiments for a microphone positioned at $x=c, y=7.9 c$ and $z=$ mid-span. Experimental acoustic measurements are shown for tripped and untripped boundary layers. For the tripped case, both the airfoil suction and pressure sides develop turbulent boundary layers. In the experiments, when the boundary layers are not tripped the suction side still develops a turbulent boundary layer due to the adverse pressure gradient. However, a laminar boundary layer is developed along the pressure side due to a favorable pressure gradient. The current numerical simulation uses the tripping mechanism along the suction side of the airfoil and, therefore, the flow configuration is similar to that from the untripped experiment. As one can observe, the present acoustic prediction shows good agreement with the experimental data for the untripped case. One can also observe the tonal noise peak in the numerical prediction due to vortex shedding from the laminar boundary layer. A similar peak is shown in the experimental measurement for the untripped configuration.

In Fig. 7, one can observe directivity plots for different frequencies for the low Mach number configuration. The directivity plots are computed for observer locations at mid-span and $7.9 c$ distant from the airfoil trailing edge. As one can observe, despite the low freestream Mach number, convection effects are important for mid- and high-frequencies. These effects are shown in Fig. 7, where one can see that acoustic pressure of high-frequency upstream lobes are amplified when mean flow effects are present in the FWH formulation. In this figure, it is important to observe the difference between the amplitude of acoustic pressure of the vortex shedding tone in Fig. 7 (b) and the amplitudes for the other frequencies. In Figs. 7 (c) and (d), the directivity plots are presented with different scales compared to Figs. 7 (a) and (b) for purposes of better visualization.

Figure 8 shows sound pressure levels for microphones positioned at $r=7.9 c, \theta=90$, 120, 150, 210, 240 and 270 and $z=$ mid-span for the $M_{\infty}=0.4$ test case. One can observe a tonal noise peak in the numerical prediction due to vortex shedding from the laminar boundary 


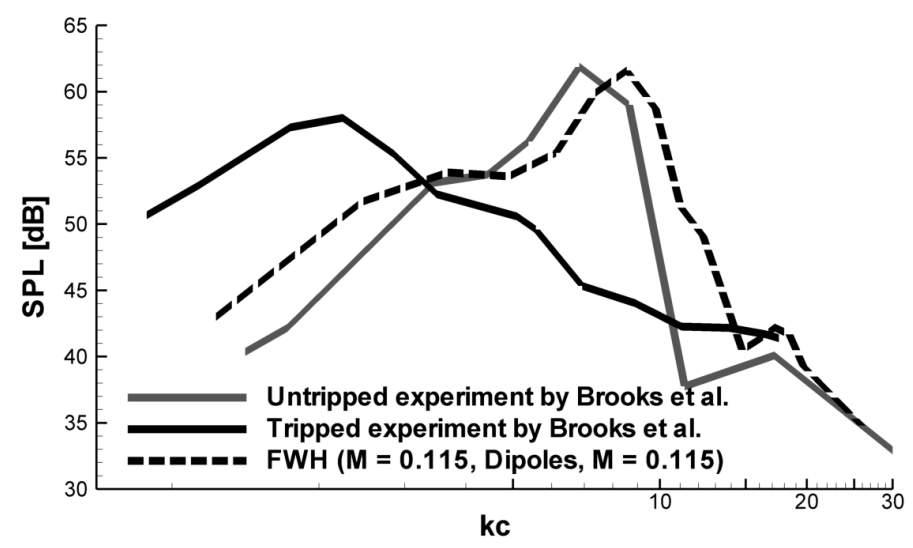

Figure 6. Sound pressure level at observer location $x=c, y=7.9 c$ and $z=$ mid-span for the $M_{\infty}=0.115$ test case.

layer at $k c \approx 27$ for all microphone locations. In the figures, one can also assess the effects of mean flow and non-linear quadrupole sources. Mean flow effects have a tendency to shift the SPL by approximately $5 \mathrm{~dB}$ for all frequencies and observer angles $\theta=90,120,240$ and 270. For observer angles $\theta=150$ and 210, mean flow effects show less pronounced effects for lower frequencies but, again, shift the SPL for higher frequencies. Quadrupole sources present significant effects at medium and high frequencies for all microphone positions. One can observe a shift of up to $5 \mathrm{~dB}$ in Fig. 8 due to quadrupole sources. When both mean flow effects and quadrupole sources are included in the FWH formulation, the SPL is shifted by up to $10 \mathrm{~dB}$ for some observer locations at medium and high frequencies, including the vortex shedding tonal peak region. Therefore, it is evident that these effects are important for the present flow configuration. Hence, while the mean flow effects increase the SPL for all frequencies for most selected observer locations, the quadrupole sources have a more pronounced effect for medium and high frequencies.

In Fig. 9, one can observe the directivity plots for different frequencies predicted at observer locations at mid-span and $7.9 c$ distant from the airfoil trailing edge. Dipole and quadrupole sources are included for both figures. The directivity plots show the effects of convection and non-linear quadrupole sources. In the upstream direction, the effects of mean flow are significant for all directivity plots shown. However, the effects of quadrupoles are not important for $k c=2.45$, but they become increasingly relevant for higher frequencies.

\section{CONCLUSIONS}

The present investigation of airfoil trailing edge noise generation and propagation concerns the broadband noise that arises from the interaction of turbulent boundary layers with the airfoil trailing edge and the tonal noise that arises from vortex shedding generated by laminar boundary layers and trailing edge bluntness. Compressible large eddy simulations (LES) are conducted for a NACA0012 airfoil with rounded trailing edge for two flow configurations with different freestream Mach numbers. The Reynolds number based on the airfoil chord is fixed at $R e_{c}=408,000$. The acoustic predictions are performed by the Ffowcs Williams \& Hawkings (FWH) acoustic analogy formulation and incorporate convective effects. Surface 




(a) $k c=2.45, S t=3.39$.

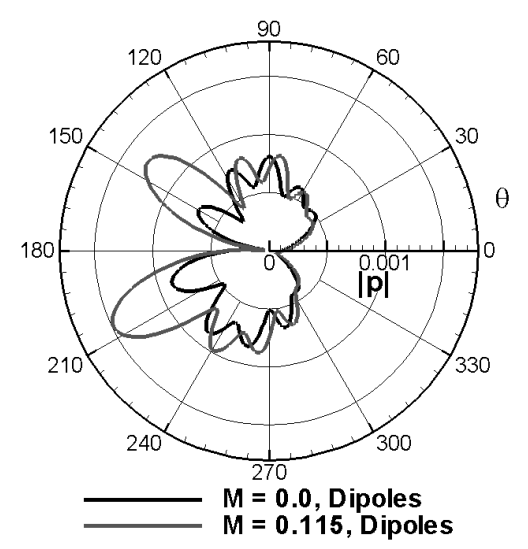

(c) $k c=14.50, S t=20.07$.

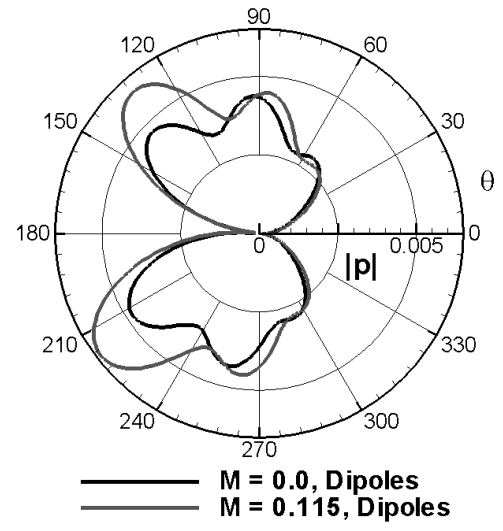

(b) $k c=8.60, S t=11.90$.

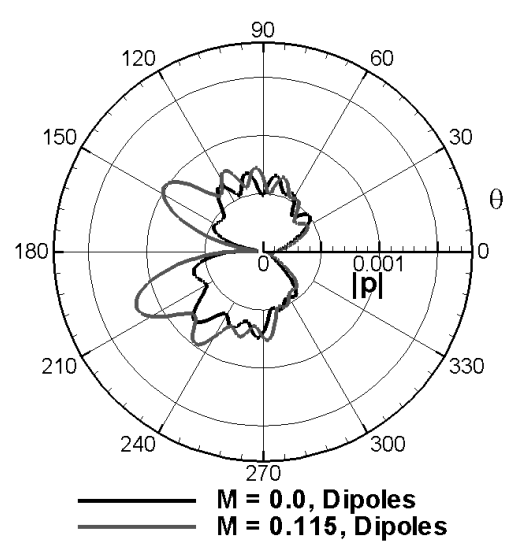

(d) $k c=18.47, S t=25.46$.

Figure 7. Directivity plots for observer locations at $r=7.9 c$ from the trailing edge for the $M_{\infty}=0.115$ test case. 


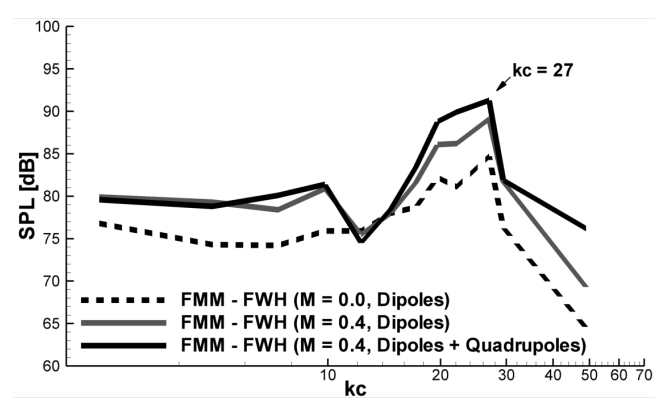

(a) $\theta=90 \mathrm{deg}$.

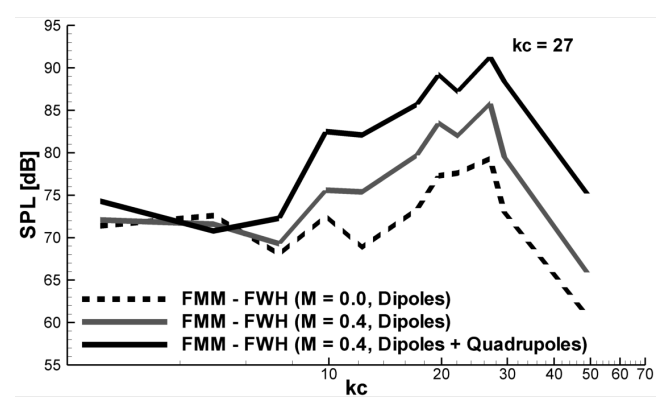

(c) $\theta=150 \mathrm{deg}$.

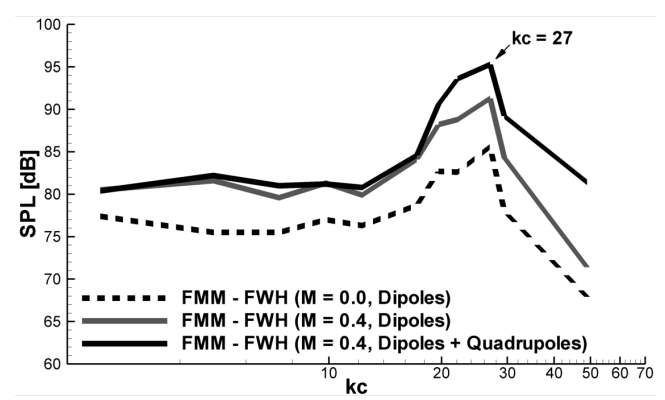

(e) $\theta=240 \mathrm{deg}$.

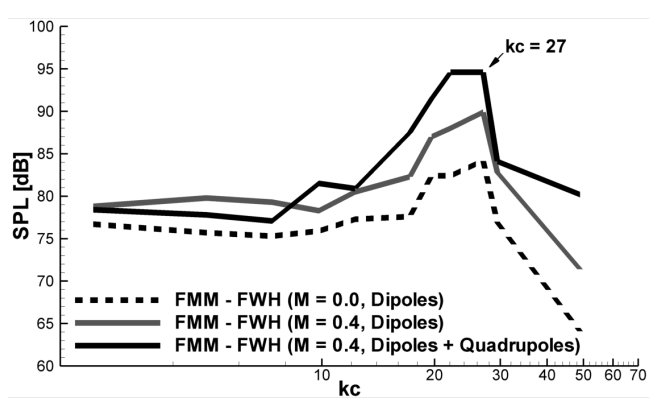

(b) $\theta=120 \mathrm{deg}$.



(d) $\theta=210 \mathrm{deg}$.

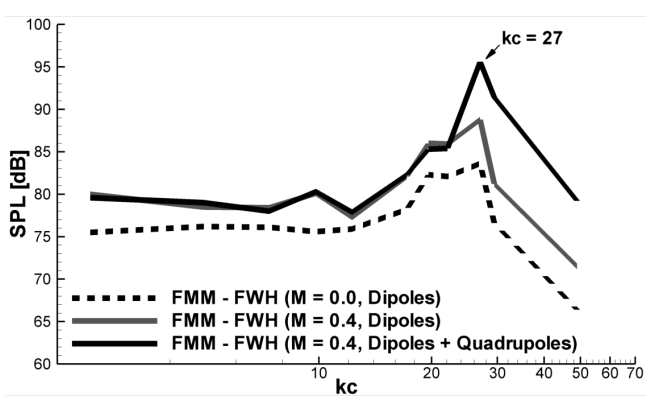

(f) $\theta=270 \mathrm{deg}$.

Figure 8. Sound pressure levels at observer locations at $r=7.9 c$ from the trailing edge for the $M_{\infty}=0.4$ test case. 


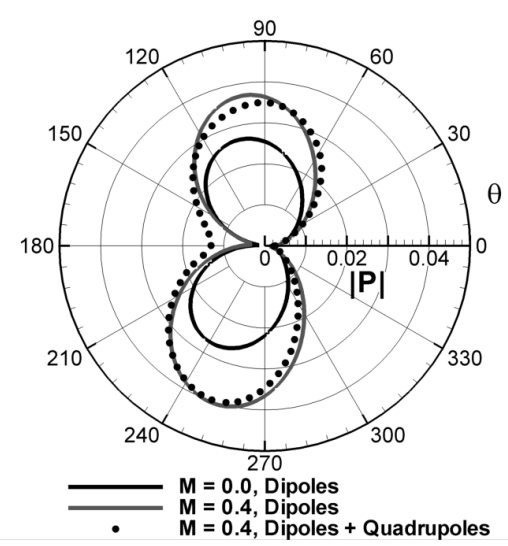

(a) $k c=2.45, S t=0.98$.

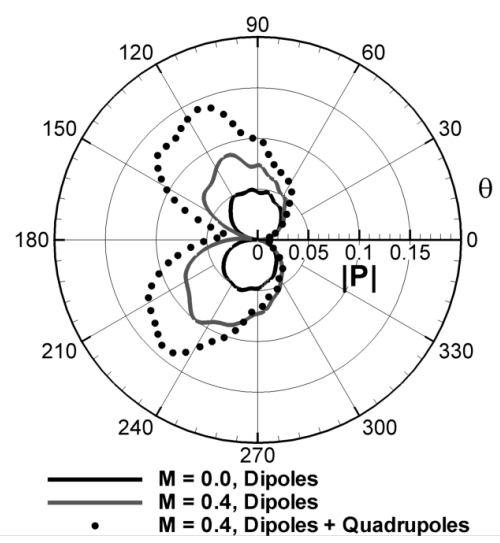

(c) $k c=19.63, S t=7.81$.

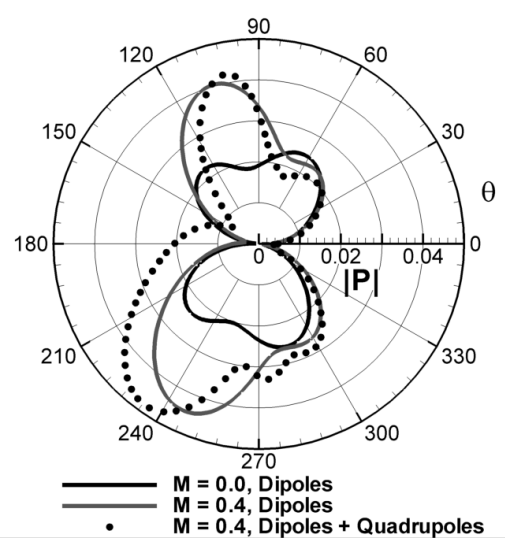

(b) $k c=4.91, S t=1.95$.

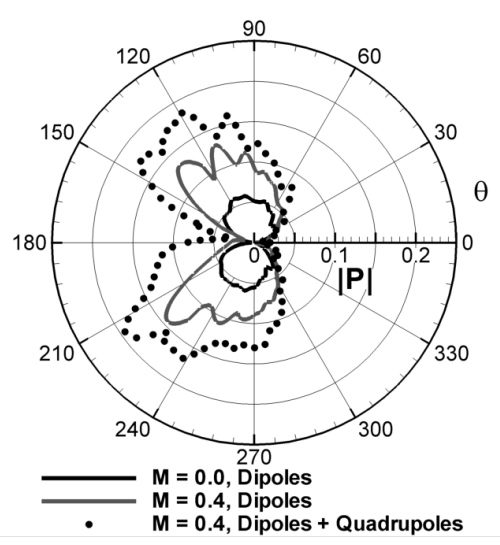

(d) $k c=26.98, S t=10.74$.

Figure 9. Directivity plots for observer locations at $r=7.9 \mathrm{c}$ from the trailing edge for the $M_{\infty}=0.4$ test case. 
and volume integrations of dipole and quadrupole source terms appearing in the FWH equation are performed using a 3D wideband multi-level adaptive fast multipole method (FMM) in order to accelerate the calculations of aeroacoustic integrals.

One of the important conclusions from the present work is that there is a significant influence of the mean flow on acoustic pressure directivity. It is typically expected that, for low speeds, there would be no important effect of convection on airfoil noise propagation. However, the work has shown that this is only true for low frequencies. The present results have demonstrated that, for medium and high frequencies, convection effects are very important even for low Mach number flows. These effects are more pronounced at upstream observation angles. For moderate Mach numbers, quadrupole sources present significant effects at medium and high frequencies. The work has also demonstrated that local scaling of the wall pressure power spectral density using boundary layer scales near the trailing edge adequately captures the changes associated with Mach number for the range analyzed. The present results also demonstrate that vortex shedding occurs due to laminar boundary layer separation. For the cases analyzed, the identification of vortex shedding effects is clear both in terms of hydrodynamic and acoustic quantities.

\section{Acknowledgements}

The authors acknowledge the financial support received from FAPESP under Grant No. 2011/12493-6, and from CNPq under Grants No. 312064/2006-3 and No. 471592/2011-0. The computational resources provided by the National Science Foundation are also gratefully acknowledged. The authors further acknowledge the following award for providing computing resources that have contributed to the research results reported within this paper: MRI-R2: Acquisition of a Hybrid CPU/GPU and Visualization Cluster for Multidisciplinary Studies in Transport Physics with Uncertainty Quantification. Finally, the authors also kindly acknowledge the financial support received from GE during the initial stages of the present work.

\section{REFERENCES}

[1] Brooks, T. F., Pope, D. S., and Marcolini, M. A., Airfoil Self-Noise and Prediction, NASA Reference Publication 1218, NASA, 1989.

[2] Sandberg, R. D., Jones, L. E., and Sandham, N. D., "Direct Numerical Simulations of Noise Generated by Turbulent Flow Over Airfoils," Proceedings of the 14th AIAA/CEAS Aeroacoustics Conference, AIAA Paper 2008-2861, 2008, pp. 1-14.

[3] Marsden, O., Bogey, C., and Bailly, C., "Direct Noise Computation of the Turbulent Flow Around a Zero-Incidence Airfoil,” AIAA Journal, Vol. 46, 2008, pp. 874-883.

[4] Sandberg, R. D., Jones, L. E., and Sandham, N. D., "Investigation and Prediction of Transitional Airfoil Self-Noise," Proceedings of the 15th AIAA/CEAS Aeroacoustics Conference, AIAA Paper 2009-3104, 2009, pp. 1-13. 
[5] Gloerfelt, X., and Le Garrec, T., "Trailing Edge Noise from an Isolated Airfoil at a High Reynolds Number," Proceedings of the 15th AIAA/CEAS Aeroacoustics Conference, AIAA Paper 2009-3201, 2009, pp. 1-26.

[6] Winkler, J., Moreau, S., and Carolus, T., "Large-Eddy Simulation and Trailing-Edge Noise Prediction of an Airfoil with Boundary-Layer Tripping," Proceedings of the 15th AIAA/CEAS Aeroacoustics Conference, AIAA Paper 2009-3197, 2009, pp. 1-25.

[7] Jones, L. E., Sandham, N. D., and Sandberg, R. D., "Acoustic Source Identification for Transitional Airfoil Flows Using Cross Correlations," AIAA Journal, Vol. 48, 2010, pp. 2299-2312.

[8] Winkler, J., Moreau, S., and Carolus, T., "Airfoil Trailing Edge Noise Prediction from Large-Eddy Simulation: Influence of Grid Resolution and Noise Model Formulations," Proceedings of the 16th AIAA/CEAS Aeroacoustics Conference, AIAA Paper 20103704, 2010, pp. 1-17.

[9] Ffowcs Williams, J. E., and Hawkings, D. L., "Sound Generation by Turbulence and Surface in Arbitrary Motion," Philosophical Transaction of the Royal Society of London, Series A, Mathematical and Physical Sciences, Vol. 264, 1969, pp. 321-342.

[10] Wolf, W. R., and Lele, S. K., "Acoustic Analogy Formulations Accelerated by Fast Multipole Method for Two-Dimensional Aeroacoustic Problems," AIAA Journal, Vol. 48, 2010, pp. 2274-2285.

[11] Wolf, W. R., and Lele, S. K., "Wideband Fast Multipole Boundary Element Method: Application to Acoustic Scattering from Aerodynamic Bodies," International Journal for Numerical Methods in Fluids, Vol. 67, 2011, pp. 2108-2129.

[12] Wolf, W. R., and Lele, S. K., "Aeroacoustic Integrals Accelerated by Fast Multipole Method," AIAA Journal, Vol. 49, 2011, pp. 1466-1477.

[13] Nagarajan, S., Lele, S. K., and Ferziger, J. H., "A Robust High-Order Method for Large Eddy Simulation,” Journal of Computational Physics, Vol. 191, 2003, pp. 392-419.

[14] Bhaskaran, R., Large Eddy Simulation of High Pressure Turbine Cascade, PhD Thesis, Stanford University, 2010.

[15] Beam, R. M., and Warming, R. F., "An Implicit Factored Scheme for the Compressible Navier-Stokes Equations," AIAA Journal, Vol. 16, 1978, pp. 393-402.

[16] Lilly, D. K., "A Proposed Modification of the Germano Subgrid-scale Closure Method," Physics of Fluids, Vol. 4, 1992, pp. 633-635.

[17] Nagarajan, S., Leading Edge Effects in Bypass Transition, PhD Thesis, Stanford University, 2004. 
[18] Lockard, D. P., "A Comparison of Ffowcs Williams-Hawkings Solvers for Airframe Noise Applications," Proceedings of the 8th AIAA/CEAS Aeroacoustics Conference, AIAA Paper 2002-2580, 2002, pp. 1-11.

[19] Wolf, W. R., and Lele, S. K., "Trailing Edge Noise Predictions Using Large Eddy Simulation and Acoustic Analogy," AIAA Journal, accepted for publication.

[20] Wolf, W. R., Airfoil Aeroacoustics: LES and Acoustic Analogy Predictions, PhD Thesis, Stanford University, 2011.

[21] Wang, M., and Moin, P., "Computation of Trailing-Edge Flow and Noise Using LargeEddy Simulation," AIAA Journal, Vol. 38, 2000, pp. 2201-2209. 